\title{
Health Promotion in Cardiac Rehabilitation Patients through the Use of a High-Intensity Interval Training Protocol
}

\author{
Michelle Tinkham \\ Cardiac Pulmonary Rehabilitation at Renker Wellness Center, Eisenhower Medical Center, Rancho Mirage, \\ CA, USA \\ Email: Michelle.Tinkham@sbcglobal.net
}

Received 1 July 2014; revised 15 August 2014; accepted 31 August 2014

Copyright @ 2014 by author and Scientific Research Publishing Inc.

This work is licensed under the Creative Commons Attribution International License (CC BY). http://creativecommons.org/licenses/by/4.0/

(c) (i) Open Access

\begin{abstract}
According to the American Heart Association's (AHA) recent statistical update, over 2150 Americans die each day from cardiovascular disease (CVD), which equals approximately 1 death every 40 seconds; many of which were under the age of 65 years old [1]. In 2009, 386,324 people, 1 in 6 Americans, died as a result of coronary artery disease (CAD) alone [1]. They also estimate 150,000 people have "silent" heart attacks each year [1]. Even though the number of cardiovascular disease deaths has declined in the last 10 years, they still accounted for $32.3 \%$ of American deaths [1]. As a result, the AHA updated their 2020 goals to improve the nation's cardiovascular health by $20 \%$ [1]. One of these methods is through the use of cardiac rehabilitation. Cardiac rehabilitation (CR) is a health promotion strategy to help return cardiac patients to their previous level of functioning, increase health, decrease comorbidities and promote education and lifestyle change. For select patients, another alternative exercise plan may exist to gain even better results. High intensity interval training (HIIT) has shown positive training results for athletes and many studies show that it may also be an effective exercise modality for many cardiac patients instead of the traditional circuit training method. This article will review current literature on the effects of HIIT on CR patients as well as a sample HIIT protocol for instituting this treatment with appropriate patients.
\end{abstract}

\section{Keywords}

Cardiac Rehabilitation, Congestive Heart Failure, High-Intensity Interval Training

\section{Introduction}

According to the American Heart Association's (AHA) recent statistical update, over 2150 Americans die each

How to cite this paper: Tinkham, M. (2014) Health Promotion in Cardiac Rehabilitation Patients through the Use of a HighIntensity Interval Training Protocol. World Journal of Cardiovascular Diseases, 4, 493-497. 
day from cardiovascular disease (CVD), which equals approximately 1 death every 40 seconds; many of which were under the age of 65 years old [1]. In 2009, 386,324 people, 1 in 6 Americans, died as a result of coronary artery disease (CAD) alone [1]. They also estimate 150,000 people have "silent" heart attacks each year [1]. Even though the number of cardiovascular disease deaths has declined in the last 10 years, they still accounted for 32.3\% of American deaths [1]. As a result, the AHA updated their 2020 goals to improve the nation's cardiovascular health by $20 \%$ [1].

According to the World Health Organization, health promotion is defined not only as increasing a person's health but also giving them control over it [2]. Due to the advancing longevity of adults in American, there is increased likelihood of the large segment of the population experiencing coronary artery disease (CAD) and being faced with many invasive treatments, as well as, complicated comorbidities in their lifetimes often robbing them of their autonomy and retirement funds. This causes a strain, not just on the person and his/her family but also monetary depletion of insurance coverage, and health care facilities struggling to deal with an increasing number of potentially fragile patients. One effective method of health promotion in cardiac patients is Cardiac Rehabilitation (CR). This program can be conducted in an in-patient or out-patient setting and comprises of education, lifestyle modification and EKG monitored exercise, tailored to the patient by CR personnel. At this time in the United States, Medicare and most insurance plans will cover CR for patients who have had a Myocardial Infarction (MI) within the last year, a new onset of Stable Angina, an Angioplasty and/or stent placement within the last six months, Coronary Artery Bypass Graft (CABG) surgery within one year, and most recently Congestive Heart Failure (CHF) [3].

\section{Purpose}

The main objective of outpatient cardiac rehabilitation (CR) programs is to improve the physiological and psychological status of persons after a coronary artery disease event, thereby reducing recovery times and future events [4]. This is achieved through monitored exercise and education to reduce risk factors, improve nutrition, manage stress and medication therapy adherence [5]. Although CR is a common recommendation now, in the past these patients were routinely placed on mobility restriction, which often led to serious decline in health, endurance and death [5]. This is based in part on the research of Dr. Herman Hellerstein who created the idea of modern day cardiac rehabilitation [6]. It has since been proven through many studies that early ambulation and individualized exercise training actually prevents complications from coronary events such as acute myocardial infarction (AMI); as well as procedures such as angioplasty and stent placement, coronary bypass graft surgery (CABG), valve repairs, and even heart transplants [6] [7].

Few changes had been made to Hellerstein's model of CR, which included moderate continuous exercise, but in recent years more attention has been given to exercise protocols that were once thought to only be valuable to athletes: high-intensity interval training (HIIT) [6]. Many studies now show that HIIT has greater benefit to CAD patients than the previously utilized moderate intensity circuit training that many CR programs, including the author's, now use. The purpose of this evidence-based project is to design a HIIT protocol based on current research that can be utilized in the author's CR program for selected appropriate patients to continue to improve outcomes.

\section{Literature Review}

A throughout internet and library search was performed for studies and guidelines focusing on the effects of HIIT and cardiovascular disease patients, specifically CAD and CHF. In order to include only the most recent evidence-based data, only research conducted within the last 5 - 10 years were included.

In Warburton et al. (2005) [8], the effectiveness of HIIT for the rehabilitation of CAD patients was studied. Fourteen men status post CABG or Angioplasty were chosen to participate. Participants were stable, average age of 56 years, average weight $190 \mathrm{lbs}$., with an aerobic capacity of 9 METs. The participants were divided into two groups. The traditional aerobic group consisted of a 10 minute warm-up, and 30 minutes of continuous exercise at $65 \%$ heart rate reserve. The HIIT group performed the same warm-up but instead performed 2 minute high-intensity sessions at $90 \%$ heart rate reserve followed by 2 minute low-intensity session at $65 \%$ heart rate reserve. At the conclusion of the 16 week study, the HIIT group demonstrated a higher treadmill to exhaustion time.

Another study focused on low-volume high-intensity exercise for patients with CAD [9]. Their study recruited 30 outpatient CR patients who had a recent CAD event. Seven patients left the study due to medication changes 
and other undisclosed reasons leaving the final sample at 22 persons. Participants were divided into two groups: moderate and high intensity. The study used a factorial repeated-measure design performing measurements before training and after 12 weeks. The study found that both groups improved aerobically but the HIT group's improvement was $5 \%$ higher than the moderate intensity group.

Steven et al. (2014) [10] conducted a study of 39 patients to evaluate the effectiveness of HHIT in cardiac rehabilitation. This study recruited both genders with a broad age range of 18 - 75 years, in a 3 day a week CR program. The group was distributed into two groups: HIIT and moderate exercise. The results found that HIIT was safe to include in standard CR and did result in greater exercise capacity and endurance of cardiovascular patients.

Since Medicare recently approved the admission of CHF patients in CR, it is also important to review studies that focus specifically on the effects of HIIT and CHF patients. Beale et al. (2013) [11] compared the traditional circuit training method with HIIT in CHF patients in an UK cardiac rehabilitation program. They recruited their 26 participants from a heart failure clinic at a local general hospital. Participants performed two 45 minute sessions for 6 weeks, half in a circuit program and the other in an interval exercise program. This study found both methods were equally effective and safe in CHF patients.

Meyer et al. (2013) [12] conducted a meta-analysis of 7 randomized studies that reviewed the effects of HIIT on chronic heart failure compared to moderate intensity exercise sessions. Participants in the studies were mostly older (54 - 75 years on average) and male. The moderate intensity programs consisted of 40 - 60 minutes of continuous exercise at $50 \%-75 \%$ heart rate reserve. The interval groups performed bouts of 30 second to 4 minutes of exercise at $70 \%-100 \%$ heart rate reserve. The reviewed studies used both cycling and treadmill as their exercise modalities. The overall conclusions from the studies reviewed were that HIIT was more effective to improve peak oxygen intake and more time effective for CHF patients.

Azevedo and Dos Santos (2014) [13] also performed a meta-analysis of 11 studies that reviewed the effects of HIIT on both CAD and congestive heart failure (CHF) patients. Seven of the studies focused specifically on CAD with training frequencies of $2-3$ times a week for a period of $10-24$ weeks total. Each study consisted of bouts of HIIT of $1-4$ minutes at $80 \%-95 \%$ heart rate reserve followed by $1-4$ minutes of recovery. The remaining four studies reviewed focused on HIIT and CHF patients at similar exercise sessions as the CAD patients. These studies showed improvements in aerobic tolerance, functional capacity, and quality of life for the participants.

In addition, the American College of Sports Medicine (ACMS) guidelines and American Association of Cardiovascular and Pulmonary Rehabilitation (AACVPR) criteria for the use of HIIT for CAD patients were also reviewed. According to the ACMS Guidelines for Exercise Testing and Prescription (2010) [14] exercise frequency should be 4 - 7 days a week, at a rate of perceived exertion (RPE) of $11-16$ and $40 \%-60 \%$ of HHR. Warm-up and cool-down should be performed for 5 - 10 minutes. Exercise sessions should be between 3 - 10 minutes with 2 - 5 minute rest periods. The AACVPR Cardiac Rehabilitation Resource Manual (2006) [15] protocol for interval training includes three minute warm-up and cool-down periods with 10 sets of 30 second exercise segments at $50 \%$ HHR followed by 1 minute rest periods.

In summary, there are numerous recent evidence-based studies that show HIIT is a safe and viable option for select CAD and CHF patients in CR to improve fitness levels. HIIT has also been shown to result in higher workload capacities in lower functioning patients, lower lactate buildup, and increased calorie expenditures [16]. In addition, interval training may also be included for patients who cannot tolerate the traditional longer sessions of moderate exercise [16].

\section{Protocol Development}

After review of multiple evidence-based studies and AACVPR and ACSM guidelines, the author discussed the parameters of the new HIIT protocol with the Medical Director of the CR program. It was determined that this protocol would only be introduced in stable patients who had been performing exercise prior to their cardiovascular event. At this time, we have not started included CHF patients in our standard CR program. The training protocol will start with a 5 - 10 minute warm-up and cool-down [8] [14]. The exercise sessions will be 30 seconds to 4 minutes at $80 \%$ - 85\% HHR with 1 - 5 minute active recovery at 50\% - 70\% HHR [5] [8] [9] [11]-[15]. The exact times will be determined by the patient's past fitness level and the AACVPR protocol already established for circuit training in CR patients. All patients will continue to be monitored by the Q-tel electrocardiogram program, blood pressure, and rate of perceived exertion (RPE) scale currently used for enrolled CR patients. The author's proposed protocol will follow at the end of the paper as Table 1. Possible limitations to this protocol may be the 
Table 1. CR high-intensity interval training (HIIT) protocol.

STATEMENT Cardiovascular exercise guidelines for the effective and safe prescription of high-intensity interval training to patients with cardiovascular disease.

Criteria: HIIT will not be applied as a standard progression for all patients, but when it is determined that it may enhance

PURPOSE patients care and/or achieving goals. Patients may be progressed to HIT when considered medically appropriate and functionally able. Patients will be reviewed by staff with Clinical Medical Director on an individual basis for consideration prior to initiating HIIT. CRRNs orientation and programming as well as ongoing progression.

MET: Metabolic Equivalent

HHR: Heart Rate Reserve: 220-age—Resting HR x desired percentage + Resting HR

DEFINITIONS RPE: Rating of Perceived Exertion on a 0 - 10 Scale will be used in all patients and will supersede heart rates in patients who report high exertion levels and will be considered as the primary indicator of volitional fatigue especially in patients who are actively taking beta-blocker medications.

Warm up/Cool down: Exercise sessions will include a 5 - 10 minute warm-up, balance drills, and a 5 - 10 minute cool-down. Cardiac transplant patients and patients with chronic angina will perform a minimum of 10 minutes warm-up and cool-down.

Mode: Initial mode will be based on patient goal, functional status, medical concerns, and desire. Sternotomy patients will avoid Cardiovascular upper body exercise forapproximately8 weeks after surgery.

Frequency: $3 \times$ wk. at CR with goal of two times a wk. additional days.

Intensity: Exercise intensity will vary based on interval being performed and will follow the below guidelines:

Peak intensity intervals: 80\% - 95\% HRR, RPE 5 - 8.

PROTOCOL Active recovery intervals: 50\% - 70\% HRR, RPE 2 - 5.

Intensity will be set based on patient tolerance, vital sign response and patient goals. Intensity will be adjusted/progressed to maintain or increase patient to within the above HRR ranges. Intensity will also be monitored with Rating of Perceived Exertion on a 1 - 10 scale and will supersede heart rate guidelines as described above.

Time: goal of 30 - $\mathbf{4 5}$ minutes of total duration including both peak and active recovery intervals.

Peak intensity intervals will range from 30 seconds to 4 minutes.

Active recovery intervals will range from 1 - 5 minutes.

Initially peak intensity intervals will be shorter duration with longer duration active recovery intervals. Interval duration will be based on patient tolerance, vital sign response and patient goals Progression will involve increasing peak intensity interval duration and/or decreasing active recovery interval duration.

low number of patients less than 65 years of age in our area, limited patients with previous exercise experience, and inability to effective ensure patients are performing the plan exactly as prescribed due to the group setting used.

\section{Conclusion}

Although many CR programs have been hesitant to include HIIT exercises to their program, evidence shows that when used appropriately, interval training can be very effective for select patients. As with any exercise program, especially with cardiovascular patients, appropriate evaluation must take place to determine safe levels and individualized modalities. A complete CR assessment by trained staff as well as agreement from the patient's cardiologist or program medical director should occur before implementing HIIT and formal exercise program.

\section{References}

[1] American Heart Association (2013) Heart Disease and Stroke Statistics--2013 Update: A Report from the American Heart Association. Circulation, 127, e6-e245. http://circ.ahajournals.org/content/127/1/e6.full.pdf+html

[2] World Health Organization (2014) Health Promotion. http://www.who.int/topics/health_promotion/en/

[3] Medicare.gov. (2014) Your Medicare Coverage. http://www.medicare.gov/coverage/cardiac-rehab-programs.html

[4] Mike, J. and Kravitz, L. (2007) Is Interval Training Safe for CAD Clients? IDEA Fitness Journal, 4, 19-21. http://www.unm.edu/ lkravitz/Article\%20folder/intervalCAD.html

[5] Mampuya, W. (2012) Cardiac Rehabilitation Past, Present and Future: An Overview. Cardiovascular Diagnosis \& Therapy, 2, 38-49. http://www.thecdt.org/issue/view/10) 
[6] Hellerstein, H. and Perry, P. (1990) Healing Your Heart. Simon and Schuster, New York.

[7] Stewart, K., et al. (2003) Cardiac Rehabilitation Following Percutaneous Revascularization, Heart Transplant, Heart Valve Surgery, and for Chronic Heart Failure. Chest, 123, 2104-2111. http://dx.doi.org/10.1378/chest.123.6.2104

[8] Warburton, D., et al. (2005) Effectiveness of High-Intensity Interval Training for the Rehabilitation of Patients with Coronary Artery Disease. American Journal of Cardiology, 95, 1080-1084. https://www.researchgate.net/publication/7894538_Effectiveness_of_high-intensity_interval_training_for_the_rehabilit ation_of_patients_with_coronary_artery_disease

[9] Currie, K., et al. (2013) Low-Volume, High-Intensity Interval Training in Patients in CAD. Medicine \& Science in Sports \& Exercise, 45, 1436-1442.

https://www.researchgate.net/publication/235884666_Low-Volume_High-intensity_Interval_Training_in_Patients_wit h_Coronary_Artery_Disease

[10] Steven, K., et al. (2014) Greater Improvement in Cardiorespiratory Fitness Using Higher-Intensity Interval Training in the Standard Cardiac Rehabilitation Setting. Journal of Cardiopulmonary Rehabilitation and Prevention, 34, 98-105. http://dx.doi.org/10.1097/HCR.0000000000000049

[11] Beale, L., McIntosh, R., Raju, P., Lloyd, G. and Brickley, G. (2013) A Comparison of High Intensity Interval Training with Circuit Training in a Short-Term Cardiac Rehabilitation Programme for Patients with Chronic Heart Failure. International Journal of Physical Medicine \& Rehabilitation, 1, 1-7. http://omicsonline.org/a-comparison-of-high-intensity-interval-training-2329-9096.1000151.pdf

[12] Meyer, P., Gayda, M., Juneau, M. and Nigam, A. (2013) High-Intensity Aerobic Interval Exercise in Chronic Heart Failure. Current Heart Failure Report, 10, 130-138. http://dx.doi.org/10.1007/s11897-013-0130-3 http://www.fondationepic.org/site/images/stories/2013_Meyer_P_HIIT_in_CHF_REVIEW.pdf

[13] Azevedo, L. and Santos, M. (2014) High-Intensity Intermittent Exercise Training for Cardiovascular Disease. Journal of Novel Physiotherapies, 4, 1-8.

http://omicsgroup.org/journals/highintensity-intermittent-exercise-training-for-cardiovascular-disease-2165-7025-199.p $\underline{\mathrm{df}}$

[14] American College of Sports Medicine (2010) ACSM’s Guidelines for Exercise Testing and Prescription. 8th Edition, LWW, Philadelphia.

[15] American Association of Cardiovascular and Pulmonary Rehabilitation (2006) AACVPR Cardiac Rehabilitation Resource Manual. Human Kinetics, Champaign.

[16] Mookerjee, S. (1998) The Application of Interval Training for Exercise Prescription in Cardiac Rehabilitation. Journal of Cardiopulmonary Rehabilitation, 18, 233-235. http://dx.doi.org/10.1097/00008483-199805000-00008 
Scientific Research Publishing (SCIRP) is one of the largest Open Access journal publishers. It is currently publishing more than 200 open access, online, peer-reviewed journals covering a wide range of academic disciplines. SCIRP serves the worldwide academic communities and contributes to the progress and application of science with its publication.

Other selected journals from SCIRP are listed as below. Submit your manuscript to us via either submit@scirp.org or Online Submission Portal.
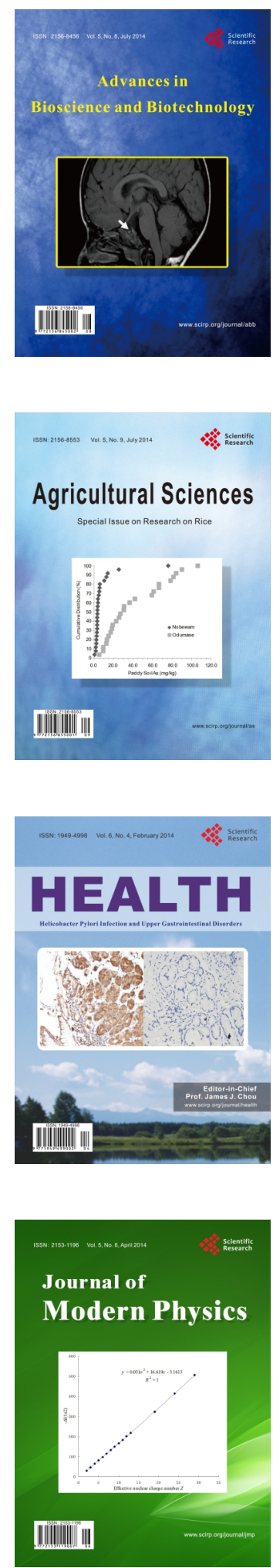
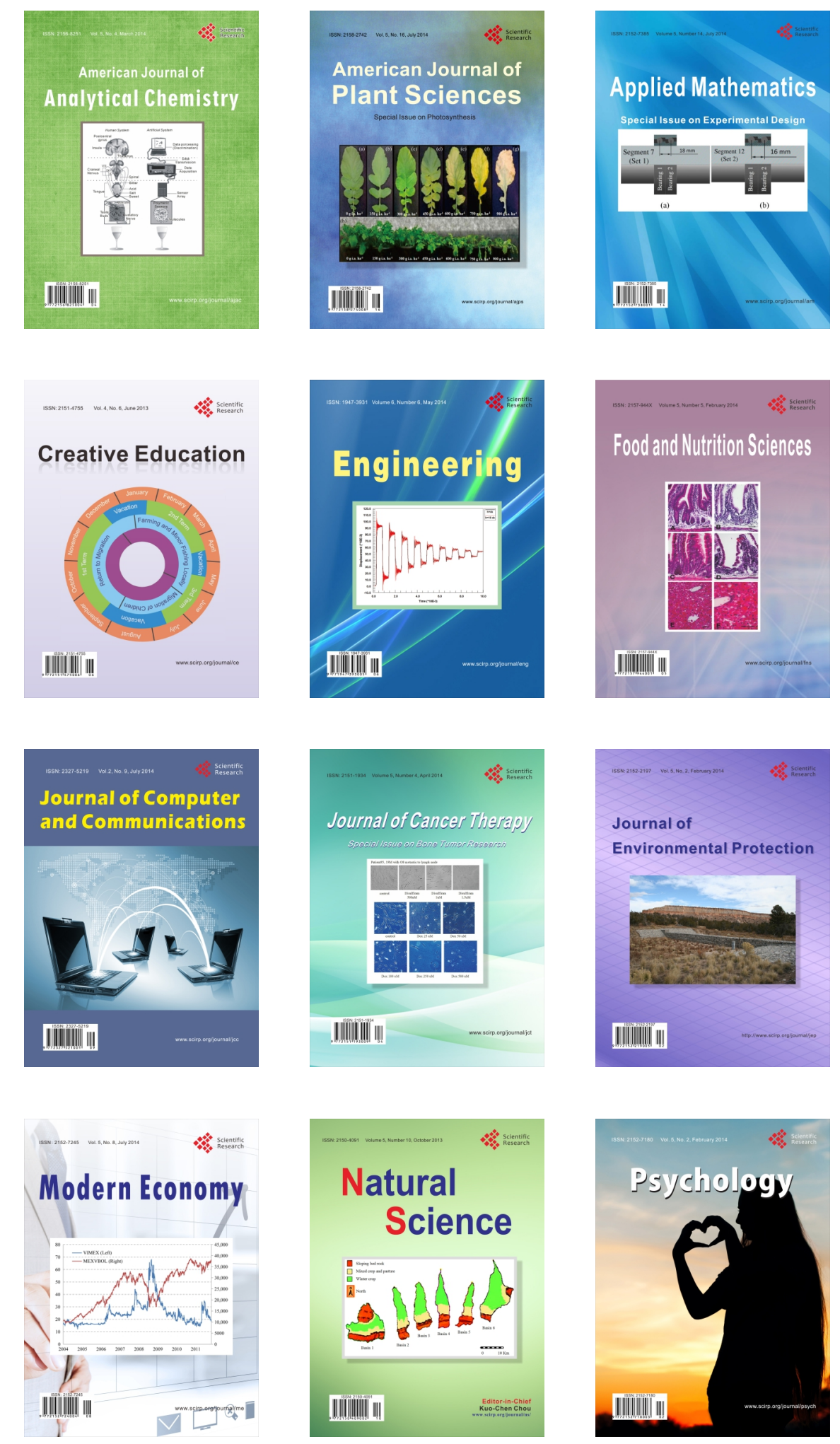